\title{
Medidas Hipertensivas em Escolares: Risco da Obesidade Central e Efeito Protetor da Atividade Física Moderada-Vigorosa
}

\author{
Hypertensive Measures In Schoolchildren: Risk Of Central Obesity And Protective Effect Of Moderate-To- \\ Vigorous Physical Activity
}

\author{
Tatiana Affornali Tozo, ${ }^{1,2}$ Beatriz Oliveira Pereira, ${ }^{1 \oplus}$ Francisco José de Menezes Junior, ${ }^{2 \oplus}$ Cristianne Morgado \\ Montenegro, ${ }^{10}$ Carla Marisa Maia Moreira, ${ }^{30}$ Neiva Leite, ${ }^{2(0)}$ \\ Universidade do Minho, ${ }^{1}$ Braga - Portugal \\ Universidade Federal do Paraná, ${ }^{2}$ Curitiba, PR - Brasil \\ Universidade do Porto, ${ }^{3}$ Porto - Portugal
}

\section{Resumo}

Fundamento: Aumento da prevalência de hipertensão arterial (HA) em crianças e adolescentes e sua associação com diversas comorbidades.

Objetivo: Verificar a associação de HA, obesidade central e obesidade geral, e nível de atividade física em escolares.

Métodos: Participaram do estudo 336 crianças e adolescentes, de 11 a 17 anos de idade. Aferiram-se estatura, peso corporal, circunferência da cintura (CC) e pressão arterial (PA). Foi calculado o índice de massa corporal escore $z$ (IMC-z). O nível de atividade física foi avaliado pela versão curta do International Physical Activity Questionnaire (IPAQ), conforme a prática em atividades físicas moderadas-vigorosas (AF-mv). Consideraram-se hipertensos os escolares que apresentaram pressão arterial sistólica (PAS) e/ou diastólica (PAD) superiores ao percentil 95, de acordo com sexo, faixa etária e estatura, ou $\geq 120 \times 80 \mathrm{mmHg}$. Utilizaram-se os testes estatísticos de $t$-Student, Qui-quadrado, Mann-Whitney e modelo de regressão logistica binária, considerando-se o nível de significância de $\mathbf{p}<0,05$.

Resultados: Foram observados que 40,5\% dos escolares apresentaram HA, 35, 11\% excesso de peso (12,5\% obesos), $13,39 \%$ CC elevada e 40,2\% foram considerados insuficientemente ativos em AF-mv. As chances de HA foram relacionadas à $C$ elevada $(O R=6,11 ; I C 95 \%: 2,59$ a 14,42) e ao excesso de peso $(O R=2,91 ; I C 95 \%: 1,76$ a 4,79). Além disso, os adolescentes que praticavam $A F-m v$ apresentaram menor risco de PAD elevada $(O R=0,33$; IC95\%:0,15 a 0,72).

Conclusão: Concluiu-se que a obesidade central, a obesidade geral e o sexo masculino foram os melhores preditores de HA em crianças e adolescentes. A prática de AF-mv demonstrou efeito protetor na PAD elevada em escolares. (Arq Bras Cardiol. 2020; 115(1):42-49)

Palavras-chave: Criança; Adolescente; Escolares; Atividade física; Circunferência da Cintura; Índice de Massa Corporal; Hipertensão; Pressão Arterial.

\section{Abstract}

Background: The proportion of arterial hypertension (AH) has increased in children and adolescents and is associated with several comorbidities.

Objective: To verify the association of arterial hypertension with central and general obesity as well as according to the level of physical activity in schoolchildren.

Methods: 336 children and adolescents aged 11 to 17 participated in the study. Height, body weight, waist circumference (WC) and blood pressure (BP) were measured. The body mass index z-score (BMI-z) was calculated. The level of physical activity was assessed by the short form of the International Physical Activity Questionnaire (IPAQ) according to the practice of moderate-to-vigorous physical activities (AF$m v)$. Students with systolic (SBP) and/or diastolic blood pressure (DBP) higher than the 95th percentile according to sex, age and height or $\geq 120 / 80$ were considered hypertensive. Statistical tests of t-Student, Chi-square, Mann-Whitney and binary logistic regression model were used, considering the significance level of $p<0.05$.

Correspondência: Tatiana Tozo •

Rua Paulino Siqueira Cortes 1986. CEP 83005-030, São José dos Pinhais, PR - Brasil

E-mail: tatinali@yahoo.com.br

Artigo recebido em 03/12/2018, revisado em 18/06/2019, aceito em 17/07/2019

DOI: https://doi.org/10.36660/abc.20180391 
Results: It was found that $40.5 \%$ of the students had AH, 35.11\% were overweight (12.5\% obese), 13.39\% had high WC and 40.2\% were considered insufficiently active in AF-mv. The chances of AH were related to high WC (OR $=6.11 ; 95 \% \mathrm{Cl}$ : 2.59 $\neg-14.42)$ and overweight $(O R=2.91 ; 95 \% \mathrm{Cl}: 1.76-4.79)$. In addition, adolescents who practiced AF-mv had a lower risk of high DBP (OR $=0.33 ; 95 \% \mathrm{Cl}$ : 0.15-0.72).

Conclusion: Central obesity was the best predictor of AH in children and adolescents, as well as general obesity and males. The practice of AFmv demonstrated a protective effect on high DBP in schoolchildren. (Arq Bras Cardiol. 2020; 115(1):42-49)

Keywords: Child; Adolescent; School Children; Physical Activity; Waist Circumference; Body Mass Index; Hipertension; Blood Pressure.

Full texts in English - http://www.arquivosonline.com.br

\section{Introdução}

A frequência de HA aumentou em todas as faixas etárias e em diversos países, ${ }^{1}$ atingindo crianças e adolescentes e tende a persistir ao longo do tempo, com a probabilidade elevada de progredir na vida adulta, ${ }^{2}$ principalmente pela crescente prevalência da obesidade, ${ }^{3}$ que está associada ao aparecimento de diversas comorbidades. ${ }^{4}$

A análise conjunta dos hábitos de vida que podem predispor ao aparecimento de doenças cardiovasculares na idade adulta tem papel importante para prevenção da hipertensão em crianças e adolescentes. ${ }^{5}$ A obesidade apresenta origem multifatorial, envolvendo aspectos do comportamento relacionados a alimentação, atividade física e fatores psicológicos. ${ }^{6}$

Portanto, o diagnóstico precoce da HA em crianças e adolescentes é relevante para se evitar o avanço da doença para a fase adulta, reduzir o risco de problemas cardiovasculares $^{7}$ e recomendar programas terapêuticos para reverter esse processo. ${ }^{8}$ Dessa forma, medidas antropométricas como o índice de massa corporal (IMC) e a CC são indicadores de baixo custo e eficientes para identificar riscos cardiovasculares. ${ }^{9} \mathrm{O}$ IMC classificado como excesso de peso demonstra obesidade geral, ${ }^{10}$ e a CC aumentada relaciona-se à obesidade central; ${ }^{11}$ esta última apresenta maior associação ao processo inflamatório em adultos e aparecimento das comorbidades cardiometabólicas. ${ }^{12}$

A mensuração da PA deve ser realizada em três ocasiões diferentes ${ }^{13}$ para confirmar o diagnóstico de HA, mas em estudos epidemiológicos geralmente é realizada em um dia e tem sido utilizado o termo "medidas hipertensivas". ${ }^{14}$ Portanto, em crianças e adolescentes, alguns estudos demonstram maiores associações entre $\mathrm{HA}$, obesidade central ${ }^{15} \mathrm{e}$ obesidade geral, ${ }^{16}$ o que gera controvérsias entre a localização da adiposidade e a PA nesta população.

Além disso, as evidências sobre a prática AF-mv e PA ainda são limitadas, bem como a relação entre medidas antropométricas e AF-mv como protetores de HA em crianças e adolescentes. Dessa forma, é importante identificar o risco de HA na adolescência para a prevenção do avanço dessa condição na vida adulta, o que pode aumentar a eficiência do tratamento. Portanto, este estudo tem como objetivo verificar a associação entre AF-mv e indicadores antropométricos de obesidade com o diagnóstico de HA em crianças e adolescentes.

\section{Métodos}

Realizou-se um estudo descritivo transversal quantitativo na cidade de São José dos Pinhais, Paraná (região Sul do Brasil). A amostragem foi constituída por conglomerados, escolhidos por conveniência (cada escola particular dos ensinos fundamental e médio da cidade foi considerada um conglomerado). Das seis instituições convidadas, localizadas na região central, somente duas escolas particulares aceitaram participar do estudo, em que foram convidados todos os escolares dos ensinos fundamental e médio.

No município, foram matriculados aproximadamente 55.289 estudantes nos níveis de ensino fundamental, nas séries finais, e ensino médio no ano de 2018. ${ }^{17}$ Estipulou-se a prevalência de $12,5 \%$ de crianças e adolescentes hipertensos na região sul do Brasil. ${ }^{18}$ Com base na seleção amostral probabilística, obteve o número total de 111 adolescentes para inferência da população estudantil na faixa etária estipulada. Incluiu-se 1,5x de indivíduos referente ao efeito de delineamento, levando-se em consideração o erro amostral de $5 \%$, sendo incluídos mais $30 \%$ em virtude de possíveis desistências, resultando em um total de 217 indivíduos entre 11 e 17 anos de idade.

Participaram do estudo 336 crianças e adolescentes voluntários, com idade de 11 a 17 anos, de ambos os sexos (173 meninas e 163 meninos). Foram excluídos do estudo gestantes, indivíduos com limitações que os impedissem de participar de algum procedimento do estudo e aqueles que não tiveram o Termo de Consentimento Livre e Esclarecido (TCLE) e o Termo de Assentimento Livre e Esclarecido (TALE) assinados. Todos os procedimentos foram aprovados pelo Comitê de Ética em Pesquisa da Pontifícia Universidade Católica do Paraná, PUC - PR, CAAE (71324017.1.0000.0020/2017.)

As medidas antropométricas foram coletadas na escola, de maneira padronizada, seguindo os procedimentos preconizados pelo Anthropometric Standartization Reference Manual. ${ }^{19}$ A estatura foi aferida por meio de um estadiômetro portátil, com resolução de 0,1 centímetro (cm), e expressa em $\mathrm{cm}$. O peso corporal foi avaliado com uma balança portátil modelo PLENA, com resolução de até 100 gramas e capacidade de $150 \mathrm{~kg}$.

Calculou-se o IMC-z, ${ }^{20}$ por meio do software WHO Anthro Plus ${ }^{\circledR}$, versão 1.0.4. Classificaram-se como eutróficos os participantes com IMC-z entre $\geq-2$ e $<+1$, com sobrepeso entre $\geq 1$ e $<2$, e obesos aqueles com $\geq 2$; de acordo com a faixa etária e o sexo, foram considerados com obesidade geral os adolescentes classificados como sobrepeso e obesidade 
(IMC-z $\geq 1$ ). Para a mensuração da CC, foi utilizada uma fita métrica inelástica, no ponto médio entre o último arco superior da crista ilíaca e a face externa da última costela. Consideraram-se com obesidade central os adolescentes com percentil $\geq 75$, de acordo com o sexo e a faixa etária. ${ }^{21}$

A aferição da PAS e da PAD seguiu as recomendações $7^{a}$ Diretriz Brasileira de Hipertensão Arterial, ${ }^{13}$ em sala de aula isolada e ambiente silencioso, utilizando-se aparelhos de pressão automáticos OMRON705-IT.22 Foram realizadas duas mensurações da PAS e da PAD no braço direito do avaliado, por enfermeiras voluntárias, com intervalo de cinco minutos entre elas, por enfermeiras voluntárias. Para a classificação de hipertensão, foram considerados os critérios de idade, sexo e percentil de estatura. ${ }^{13}$ Deste modo, valores abaixo do percentil 90 foram considerados adequados (normotensos), desde que inferiores a $120 \times 80 \mathrm{mmHg}$, crianças com percentis entre 90 e 95 foram enquadradas como pré-hipertensas (limítrofe) e com percentil igual ou superior a 95 foram consideradas hipertensas.

O nível de atividade física foi avaliado pelo IPAQ. ${ }^{23}$ As perguntas avaliadas referem-se às atividades físicas praticadas na semana anterior à aplicação do questionário. Classificaramse os escolares como suficientemente ativos (ativos ou muito ativos) ou insuficientemente ativos (irregularmente ativos A e B ou sedentários). ${ }^{23}$

\section{Análise estatística}

A análise estatística foi realizada pelo software Statistical Package for the Social Science (SPSS), versão 24. A normalidade dos dados foi aferida pelo teste Shapiro-Wilk. Para comparação entre sexos, estado nutricional, classificação da CC e AF-mv foram utilizados o teste $t$-Student independente para as variáveis paramétricas e o teste de Mann-Whitney para as não paramétricas. Utilizou-se o teste Qui-quadrado para avaliar as proporções entre os escolares considerados adequados, pré-hipertensos e hipertensos, de acordo com valores de PAS e PAD elevados. A análise de odds ratio foi usada para indivíduos considerados adequados e hipertensos, a partir das variáveis antropométricas e da AF-mv, por meio de regressão logística binária. Considerou-se o nível de significância de $\mathrm{p}<0,05$ para todas as análises.

\section{Resultados}

O excesso de peso foi encontrado em 35,11\% dos 336 escolares avaliados, sendo 12,5\% classificados como obesos. Foram classificados com obesidade central $13,39 \%$ dos escolares, 59,8\% como suficientemente ativos em práticas de AF-mv e 52,97\% apresentaram PA elevada, sendo $12,5 \%$ pré-hipertensos e 40,5\% hipertensos. A distribuição da amostra de acordo com o sexo e a faixa etária está apresentada na Tabela 1.

Observou-se que os valores médios de PAS $(p<0,001)$, IMC-z $(p=0,034)$, IMC $(p=0,001)$ e CC $(p<0,001)$ foram maiores em meninos do que em meninas. Por outro lado, as meninas apresentaram valores superiores de PAD $(p=0,009)$. Além disso, meninos participaram por mais tempo de atividades físicas leves a vigorosas do que meninas $(p=0,007$; $p=0,009$ ) (Tabela 2).
Tabela 1 - Distribuição da amostra por sexo e faixa etária

\begin{tabular}{lcc}
\hline Faixa etária (anos) & Sexo masculino $(\mathrm{n})$ & Sexo feminino $(\mathrm{n})$ \\
\hline 12 & 33 & 29 \\
13 & 38 & 29 \\
14 & 34 & 36 \\
15 & 23 & 36 \\
16 & 21 & 29 \\
17 & 14 & 15 \\
\hline
\end{tabular}

$\mathrm{Na}$ amostra total, identificou-se que os indivíduos com obesidade geral apresentaram maiores valores de PAS $(p<0,001)$ e estatura- $z(p=0,005)$ do que eutróficos (Tabela 3 ). De acordo com o sexo, observou-se que tanto meninos quanto meninas com obesidade geral apresentaram maiores valores de PAS $(p=0,003 ; p=0,001)$ e CC $(p<0,001 ; p<0,001)$, entretanto somente as meninas apresentaram maiores valores de estatura-z e maior participação em atividades leves e vigorosas em comparação às eutróficas $(p<0,05)$.

No grupo com obesidade central, as meninas apresentaram maiores valores de PAS $(p=0,026)$, peso corporal $(p<0,001)$, estatura $(p=0,038)$ e IMC-z $(p<0,001)$ em comparação ao grupo considerado adequado. Em relação aos meninos, o grupo com obesidade central apresentou maiores valores de PAS $(p=0,002), \operatorname{PAD}(p=0,003), I M C(p<0,001)$ e IMC$(p<0,001)$ em comparação ao grupo adequado (Tabela 4). Entretanto, não foram identificadas diferenças para a prática de atividade física.

As medidas antropométricas e de PA também foram avaliadas de acordo com a AF-mv (Tabela 5). Na amostra total, os indivíduos ativos apresentaram maiores valores de peso corporal $(p=0,002)$, IMC- $z(p=0,016)$, estatura- $z$ $(p=0,001)$, além da PAD mais baixa $(p=0,046)$ em relação aos classificados como insuficientemente ativos. Em relação às meninas consideradas suficientemente ativas, foram identificados maiores valores para IMC $(p=0,005)$, estatura $(p=0,048), C C(p=0,015)$, IMC-z $(p=0,005)$ e estatura- $Z$ $(p=0,016)$ em relação às insuficientemente ativas. Os meninos suficientemente ativos possuíam estatura-z maior $(p=0,025)$ em relação aos insuficientemente ativos.

Analisando-se os parâmetros antropométricos CC, IMC-z e AF-mv com a PA (Tabela 6), os meninos apresentaram maior proporção de pré- hipertensão e hipertensão arterial $(p=0,033)$. Os indivíduos considerados ativos apresentaram maior proporção de pré-hipertensão diastólica, e os sedentários apresentaram maior proporção de $\mathrm{HA}(p=0,015)$. Constatou-se que os adolescentes com obesidade central e aqueles com excesso de peso apresentaram maior proporção de pré-hipertensão e hipertensão e PAS elevada $(p<0,001)$.

Segundo a análise de risco de chance (Tabela 7), não houve diferença entre os sexos para $\mathrm{HA}(\mathrm{OR}=1,40$; IC 0,88 a 2,22), PAS (OR=1,35; IC 0,85 a 2,14) e PAD (OR=0,84; IC 0,40 a 1,77 ) elevadas. Por outro lado, de acordo com os indicadores de adiposidade, os indivíduos com obesidade central apresentaram 6,11 (IC 2,59 a 14,42) vezes mais chances de ter $\mathrm{HA}$ em relação àqueles com $\mathrm{CC}$ adequadas, e os indivíduos com obesidade geral apresentaram 2,91 $(\mathrm{IC}=1,77=4,79)$ vezes mais em relação aos que apresentaram IMC-escore $\mathrm{z}$ adequado. Além disso, foi observado que os 
Tabela 2 - Caraterísticas da amostra

\begin{tabular}{|c|c|c|c|c|}
\hline & $\begin{array}{c}\text { Total } \\
(n=336)\end{array}$ & $\begin{array}{l}\text { Sexo feminino } \\
\quad(n=173)\end{array}$ & $\begin{array}{l}\text { Sexo masculino } \\
\qquad(n=163)\end{array}$ & $p$ \\
\hline Idade (anos)\# & $14,50[13,35$ a 15,88$]$ & $14,74[13,52$ a 15,99$]$ & $14,35[13,16$ a 15,54$]$ & 0,103 \\
\hline Peso corporal (kg)\# & $56,25[47,90$ a 64,60$]$ & $54,70[46,90$ a 62,00$]$ & $58,90[49,35$ a 70,50$]$ & 0,001 \\
\hline Estatura (cm) & $1,62[0,10]$ & $1,59[0,07]$ & $1,66[0,10]$ & $<0,001$ \\
\hline Estatura-z\# & $0,49[0,29$ a 0,78$]$ & $0,44[0,26$ a 0,71$]$ & $0,57[0,34$ a 0,82$]$ & 0,003 \\
\hline CC (cm)\# & $68,00[62,50$ a 74,00$]$ & $65,00[60,00$ a 70,00$]$ & $71,00[65,50$ a 78,00$]$ & $<0,001$ \\
\hline IMC $\left(\mathrm{kg} / \mathrm{m}^{2}\right) \#$ & $21,29[19,00$ a 24,09$]$ & $21,55[19,17$ a 24,01$]$ & $20,79[18,87$ a 24,80$]$ & 1,000 \\
\hline IMC-z & $0,51[1,22]$ & $0,37[1,16]$ & $0,65[1,27]$ & 0,034 \\
\hline PAS (mmHg)\# & $124,00[113,50$ a 132,50$]$ & $120,00[111,00$ a 131,00$]$ & $127,00[118,00$ a 135,25$]$ & $<0,001$ \\
\hline PAD (mmHg)\# & $69,50[63,38$ a 75,00$]$ & $70,00[65,00$ a 76,50$]$ & $68,00[61,75$ a 74,00$]$ & 0,009 \\
\hline AF-Mod (min/dia)\# & $17,14[6,43$ a 34,64$]$ & $12,86[6,43$ a 34,29$]$ & $17,14[6,79$ a 37,14$]$ & 0,282 \\
\hline AF-Vig (min/dia)\# & $7,14[0,00$ a 17,14$]$ & $5,71[0,00$ a 14,29$]$ & $8,57[0,00$ a 25,71$]$ & 0,009 \\
\hline
\end{tabular}

DP: desvio padrão; \#não paramétricas; PAS: pressão arterial sistólica; PAD: pressão arterial diastólica; IMC: índice de massa corporal; CC: circunferência da cintura; IMC-z: IMC-escore Z; AF-L: atividade física leve; AF-Mod: atividade física moderada; AF-Vig: atividade física vigorosa.

adolescentes suficientemente ativos apresentaram redução de aproximadamente $1 / 3$ do risco de PAD elevada $(\mathrm{OR}=0,33$; IC95\%: 0,15 a 0,72).

\section{Discussão}

Os principais resultados revelaram maior risco para $\mathrm{HA}$ em escolares com obesidade abdominal $(\mathrm{OR}=6,11)$ e obesidade geral $(O R=2,91)$. Além disso, adolescentes que praticam $A F-$ mv apresentaram redução de 33\% do risco de PAD elevada. A literatura atual tem sido consistente em demonstrar que o IMC-z e a CC estão fortemente associados com HA na infância e na adolescência. ${ }^{9}$ Em acréscimo, os achados deste estudo exibem relevante fator protetivo da prática de AF-mv para a HA na adolescência, aspecto que foi pouco explorado em estudos populacionais.

De acordo com todo o exposto, as medidas antropométricas representam significativos preditores de HA, que se justificam como alternativa simples, rápida, de fácil interpretação e de bom custo-efetividade. ${ }^{24,25}$ Vários relatos demonstram associação entre PA, IMC e CC, sugerindo a obesidade como forte fator de risco para o desenvolvimento de HA na vida adulta. ${ }^{9,16} \mathrm{~A}$ distribuição excessiva de gordura visceral é acompanhada por alterações de vários marcadores inflamatórios e endoteliais ${ }^{26}$ que estimulam o aumento de eventos de resistência à insulina, a disfunção endotelial e a elevação da retenção de líquido, que podem promover variações nos níveis de PA e crescimento do risco cardiovascular. ${ }^{27}$

Identificou-se que $40,5 \%$ dos adolescentes apresentaram $\mathrm{HA}$, sendo metade dos escolares com excesso de peso e 2/3 com CC elevada foram diagnosticados como HA em maior proporção em relação a adolescentes com medidas adequadas. Em estudo de representatividade nacional e regional que avaliou 73.399 estudantes com idades entre 12 e 17 anos da região Sul do Brasil, estimou-se que a prevalência de HA foi de $12,5 \%$ e de pré-hipertensão foi de
$17 \%$, o excesso de peso variou entre $29,8 \%{ }^{1}$ e $35,5 \%{ }^{28}$ dos adolescentes sul-brasileiros. Sugere-se que, além dos fatores genéticos e ambientais, a obesidade e a HA podem estar relacionadas às disfunções metabólicas. ${ }^{27}$ Com relação às diferenças entre sexos, foram identificadas prevalência de HA e PAS similares entre meninos e meninas, entretanto, as meninas apresentaram maiores médias de PAD. Resultados semelhantes foram encontrados na literatura. ${ }^{9}$ Uma possível explicação deve-se ao fato de que as meninas praticam menos atividades físicas por dia em relação aos meninos, o que demonstrou efeito protetivo para PAD elevada.

Além disso, observou-se que meninas com excesso de peso praticam maior tempo de atividades físicas, bem como adolescentes considerados ativos apresentaram maiores médias de indicadores antropométricos. Este dado pode refletir a participação em atividades físicas como uma estratégia para reduzir o peso corporal. ${ }^{14}$

Identificou-se que altos valores de CC e IMC-z foram associados ao maior risco de $\mathrm{HA}$, entretanto, os considerados suficientemente ativos apresentaram redução de $1 / 3$ do risco de PAD elevada, o que sugere que AF-mv pode interferir nos níveis pressóricos, além de reduzir o risco metabólico. ${ }^{29}$ Entretanto, outro estudo ${ }^{30}$ demonstrou que somente o sobrepeso e a obesidade associaram-se diretamente com HA, mas não a prática de AF-mv. ${ }^{31}$ Grande parte dos adolescentes foram considerados suficientemente ativos, o que pode estar relacionado ao nível socioeconômico, com a oferta de atividades físicas fora dos períodos escolares. ${ }^{32}$

O processo de urbanização, os avanços tecnológicos da sociedade moderna e o aumento da violência estão associados com a mudança no comportamento de crianças e adolescentes. ${ }^{33} \mathrm{O}$ aumento do tempo em atividades sedentárias e a menor prática AF-mv favorecem o ganho de peso e as doenças associadas à obesidade, entre elas, a HA. ${ }^{1}$ Recomenda-se a prática de no mínimo de 300 minutos de AFmv por semana para fornecer benefícios adicionais de saúde. ${ }^{34}$ 


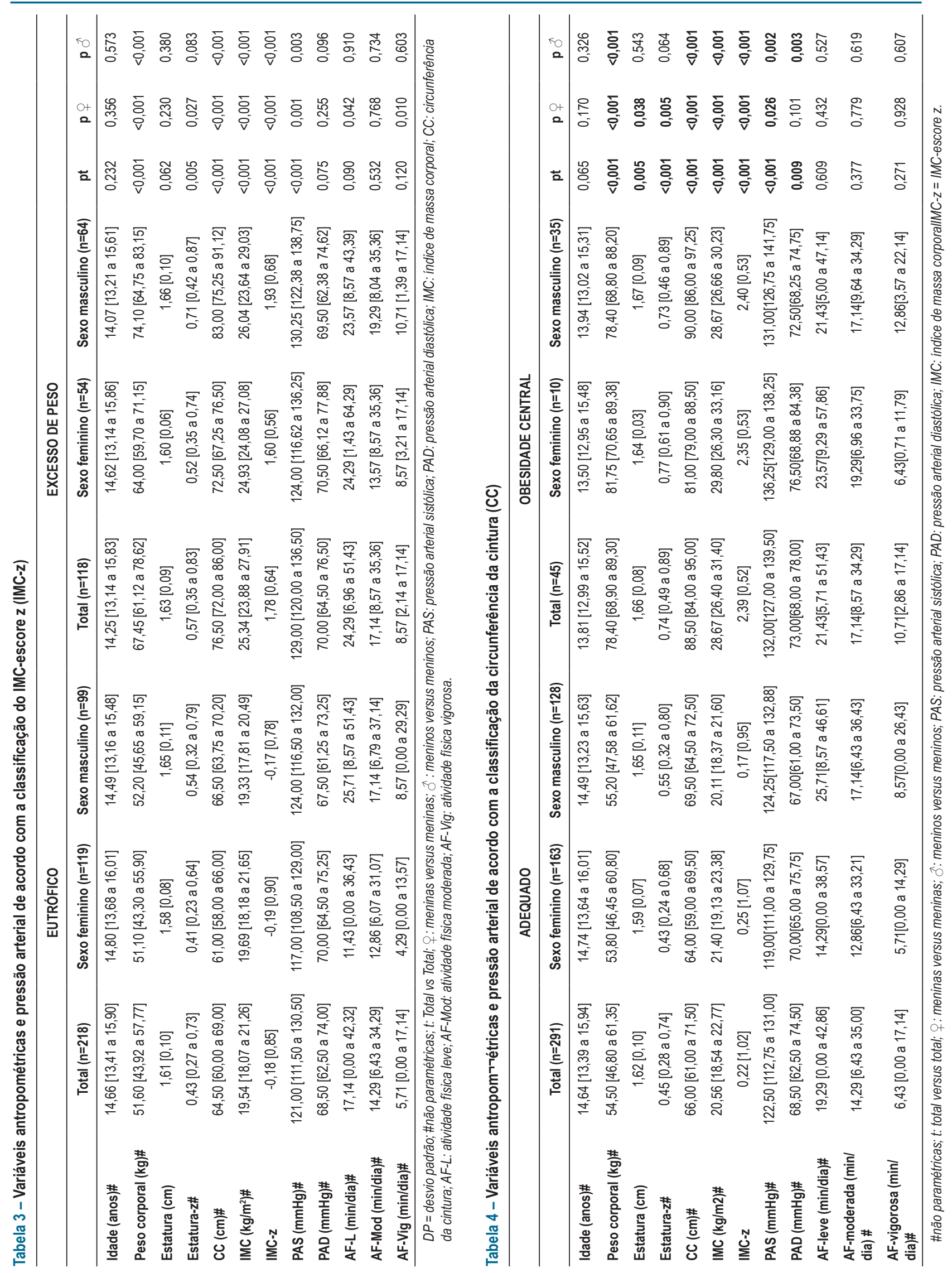




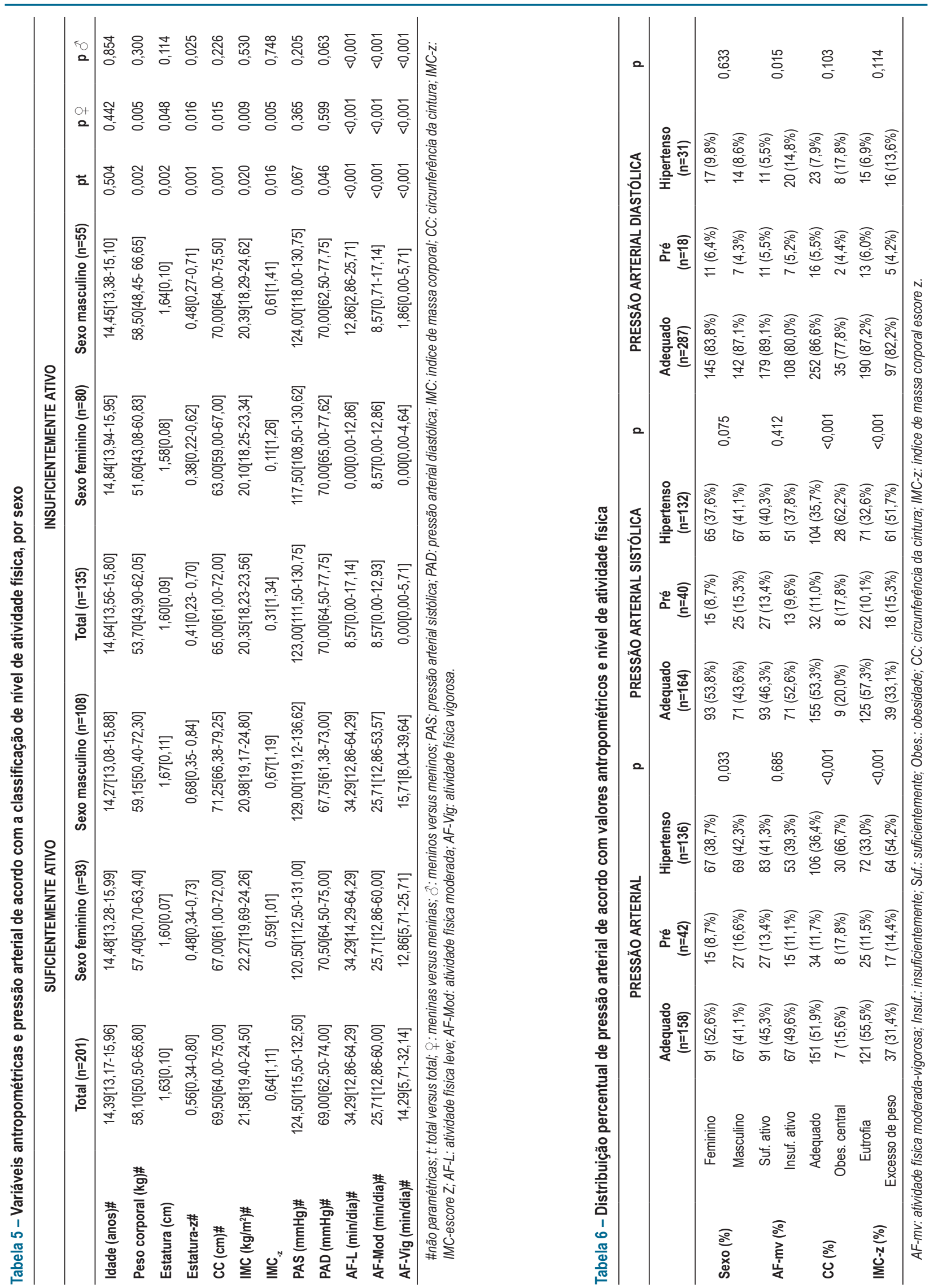


Tabela 7 - Odds ratios (OR) para o risco de pressão arterial elevada entre as variáveis antropométricas e o nível de atividade física

\begin{tabular}{lccc}
\hline & $\begin{array}{c}\text { HA } \\
\text { OR (IC95\%) }\end{array}$ & $\begin{array}{c}\text { PAS elevada } \\
\text { OR (IC95\%) }\end{array}$ & $\begin{array}{c}\text { PAD elevada } \\
\text { OR (IC95\%) }\end{array}$ \\
\hline Sexo masculino & $1,40(0,88$ a 2,22$)$ & $1,35(0,85$ a 2,14$)$ & $0,84(0,40$ a 1,77$)$ \\
AF-mv (suficientemente ativo) & $1,15(0,72$ a 1,84) & $1,21(0,76$ a 1,93) & $0,33(0,15$ a 0,72$)$ \\
CC (obesidade central) & $6,11(2,59$ a 14,42) & $4,64(2,10$ a 10,23) & $2,50(1,04$ a 6,03$)$ \\
IMC-z (Obesidade geral) & $2,91(1,76$ a 4,79) & $2,75(1,68$ a 4,52) & $2,09(0,99$ a 4,40) \\
\hline
\end{tabular}

AF-mv: atividade física moderada-vigorosa; CC: circunferência da cintura; IMC-z: índice de massa corporal escore z; há: hipertensão arterial; PAS: pressão arterial sistólica; PAD: pressão arterial diastólica; IC: intervalo de confiança; valores de $p$ com nível de significância $p<0,005$.

Neste sentido, o aspecto de relevância encontrado neste estudo refere-se à associação entre valores mais baixos de PAD em adolescentes que praticam AF-mv, sugerindo que essa prática possa interferir nos níveis pressóricos na população infantojuvenil. ${ }^{30}$ Estudo recente mostrou que adolescentes com melhores aptidões musculares exibiram menores níveis de PAD. ${ }^{35}$ Por todos os motivos expostos, conclui-se que intervenções que estimulem a transição da inatividade física para um estágio de ação promovem impactos imediatos para o aumento da prática da atividade física em escolares, ${ }^{36}$ o que pode ser considerado fator de proteção para HA.

Portanto, a eficácia em detectar precocemente fatores de risco pode contribuir para prevenção de doenças cardiovasculares na vida adulta, apesar de mudanças de hábitos e atitudes representarem tarefas complexas e muitas vezes de resultados insatisfatórios. Entretanto, políticas de saúde direcionadas para escolares, assim como investimentos sociais para melhoria da prática de AF-mv, poderão no futuro determinar mudanças significativas da população. Neste sentido, é de grande importância que profissionais da educação e da saúde promovam o controle e a prevenção da HA, assim como de outros fatores de risco associados a doenças cardiovasculares.

Este estudo apresenta algumas limitações como o pequeno número da amostra e a quantidade de vezes que a PA foi aferida (deve ser realizada em pelo menos três ocasiões distintas para melhor diagnosticar os escolares hipertensos). Outra limitação é o uso de questionário recordatório para avaliar o nível de atividade física, porém o IPAQ apresenta excelente associação com AF-mv. ${ }^{23}$ Também não foram verificadas as variáveis socioeconômicas, maturação sexual, ingestão de sal da dieta e antecedentes familiares para HA. Entretanto, vale ressaltar que o ponto forte foi associar a $\mathrm{HA}$ ao diagnóstico de obesidade central e obesidade geral, bem como destacar a importância da prática de AF-mv como fator de proteção às alterações na PA em crianças e adolescentes. Tais avaliações são importantes como forma preventiva em saúde pública, pois muitas crianças e adolescentes não apresentam a oportunidade de terem a PA avaliada na escola.

\section{Conclusões}

Neste estudo, observou-se que metade dos escolares avaliados apresentaram HA e 1/3 obesidade geral. Além disso, as medidas antropométricas de CC e IMC-z foram significativamente relacionadas ao maior risco de $\mathrm{HA}$, e a prática de atividades físicas aparece como fator preventivo de PAD elevada em crianças e adolescentes. Desta forma, sugere-se a implantação de programas que estimulem o estilo de vida saudável no ambiente escolar, para contribuir com a redução dos indicadores de obesidade central e obesidade geral, bem como prevenir contra HA ao aumentar a prática de AF-mv na população infantojuvenil.

\section{Agradecimentos}

Este trabalho foi apoiado pela Coordenação de Aperfeiçoamento de Pessoal de Nível Superior (CAPES), pelo Conselho Nacional de Desenvolvimento Científico e Tecnológico (CNPq), pelo Centro de Investigação em Estudos da Criança (CIEC), pelo Projeto Estratégico UID/ CED/00317/2013, por meio dos Fundos Nacionais da Fundação para a Ciência e a Tecnologia (FCT), cofinanciado pelo Fundo Europeu de Desenvolvimento Regional (FEDER) por meio do COMPETE 2020 - Programa Operacional Competitividade e Internacionalização (POCl) com a referência POCl-01-0145FEDER-007562.

\section{Contribuição dos Autores}

Concepção e desenho da pesquisa: Tozo TA, Pereira BO, Moreira CMM, Leite N; Obtenção de dados: Tozo T; Análise e interpretação dos dados: Tozo T, Pereira BO, Menezes Junior FJ, Montenegro CM, Moreira CMM, Leite N; Análise estatística: Menezes Junior FJ; Obtenção de financiamento: Pereira BO, Moreira CMM, Leite N; Redação do manuscrito: Tozo T, Pereira BO, Menezes Junior FJ, Montenegro CM; Revisão crítica do manuscrito quanto ao conteúdo intelectual importante: Pereira $\mathrm{BO}$, Leite $\mathrm{N}$.

\section{Potencial conflito de interesses}

Declaro não haver conflito de interesses pertinentes.

\section{Fontes de financiamento}

O presente estudo foi financiado pela Coordenação de Aperfeiçoamento de Pessoal de Nível Superior (CAPES), Conselho Nacional de Desenvolvimento Científico e Tecnológico (CNPq), Centro de Investigação em Estudos da Criança (CIEC), pelo Projeto Estratégico UID/CED/00317/2013, por meio dos Fundos Nacionais da Fundação para a Ciência e a Tecnologia (FCT), cofinanciado pelo Fundo Europeu de Desenvolvimento Regional (FEDER) por meio do COMPETE 2020 - Programa Operacional Competitividade e Internacionalização ( $\mathrm{POCl}$ ) com a referência POCI-01-0145-FEDER-007562.

\section{Vinculação acadêmica}

Este artigo é parte da tese de doutorado de Tatiana Affornali Tozo Universidade do Minho em regime de Cotutela com a Universidade Federal do Paraná. 


\section{Referências}

1. Bloch KV, Klein CH, Szklo M, Kuschnir MC, Abreu GA, Barufaldi LA, et al. ERICA: prevalences of hypertension and obesity in Brazilian adolescents. Rev Saude Publica. 2016;50(Suppl 1):1s-12s.

2. Horowitz B, Miskulin D, Zager P. Epidemiology of hypertension in CKD. Adv Chronic Kidney Dis. 2015;22(2):88-95.

3. GBD 2015 Obesity Collaborators, Afshin A, Forouzanfar MH, Reitsma MB, Sur P, Estep K, et al. Health effects of overweight and obesity in 195 countries over 25 years. N Engl J Med. 2017;377(1):13-27.

4. Lobstein T, Jackson-Leach R. Planning for the worst: estimates of obesity and comorbidities in school-age children in 2025. Pediatr Obes. 2016;11(5):321-5

5. Soares R, Tosta LS, Cavalcante LR, Zarife AS, Brito LL, Fraga-Maia H. Cardiovascular risk factors associated with hypertension in children and adolescent students.. J Phys Res. 2018;8(4):478-88.

6. Lee EY, Yoon $\mathrm{KH}$. Epidemic obesity in children and adolescents: risk factors and prevention. Front Med. 2018;12(6):658-66.

7. Chen W, Srinivasan SR, Ruan L, Mei H, Berenson GS. Adult hypertension is associated with blood pressure variability in childhood in blacks and whites: the bogalusa heart study. Am J Hypertens. 2011;24(1):77-82.

8. Kelly RK, Magnussen CG, Sabin MA, Cheung M, Juonala M. Development of hypertension in overweight adolescents: a review. Adolesc Health Med Ther. 2015 Oct $21 ; 6: 171-87$

9. Manios Y, Karatzi K, Protogerou AD, Moschonis G, Tsirimiagou C, Androutsos $\mathrm{O}$, et al. Prevalence of childhood hypertension and hypertension phenotypes by weight status and waist circumference: the Healthy Growth Study. Eur J Nutr. 2018;57(3):1147-55.

10. Ferreira F, Mota JA, Duarte J. Prevalence of overweight and obesity in school adolescents of Castelo Branco district, Portugal: a study based in body mass index, waist circumference and percentage of body fat. Rev Port Saúde Pública. 2012;30(1):47-54.

11. Shuster A, Patlas M, Pinthus JH, Mourtzakis M. The clinical importance of visceral adiposity: a critical review of methods for visceral adipose tissue analysis. Br J Radiol. 2012;85(1009):1-10.

12. Kuschnir MCC, Bloch KV, Szklo M, Klein CH, Barufaldi LA, Abreu GA, et al. ERICA: Prevalence of metabolic syndrome in Brazilian adolescents. Rev Saúde Pública. 2016;50(Suppl 1):1s-13s.

13. Malachias M, Souza W, Plavnik FL, Rodrigues CIS, Brandão AA, Neves MFT, et al. 7a Diretriz Brasileira de Hipertensão Arterial. Arq Bras Cardiol. 2016;107(3 Supl 3):1-83.

14. Leite N, Moser DC, Góes SM, Fabricio C, Milano GE, Stefanello JMF. High blood pressure and overweight in students from public schools of Curitiba (Paraná State, Brazil). Fisioter Mov. 2009;22(4):477-87.

15. Beck CC, Lopes AS, Pitanga FJG. Anthropometric Indicators as Predictors of High Blood Pressure in Adolescents.. Arq Bras Cardiol. 2011;96(2):126-33.

16. Moser DC, Giuliano Ide C, Titski AC, Gaya AR, Coelho-e-Silva MJ, Leite N. Anthropometric measures and blood pressure in school children. J Pediatr (Rio J). 2013;89(3):243-9.

17. Instituto Paranaense de Desenvolvimento Econômico e Social. Município de São Miguel do Iguaçu Fevereiro 2019. Curitiba: IPARDES; 2019.

18. Bloch KV, Cardoso MA, Sichieri R. Study of cardiovascular risk factors in adolescents (ERICA): results and potentiality. Rev Saúde Pública. 2016;50(Suppl 1):16-8.
19. Lohman TG, Roche AF, Martorell R. Anthropometric standardization reference manual. Human Kinetics Books. Champaign, IL: Human Kinetics Books; 1988. $177 \mathrm{p}$.

20. de Onis M. WHO Child Growth Standards. Rev Chil Pediatr. 2009;80(4):379-80.

21. Fernández JR, Redden DT, Pietrobelli A, Allison DB. Waist circumference percentiles in nationally representative samples of African-American, European-American, and Mexican-American children and adolescents. J Pediatr. 2004;145(4):439-44.

22. Stergiou GS, Yiannes NG, Rarra VC. Validation of the Omron 705 IT oscillometric device for home blood pressure measurement in children and adolescents: the Arsakion School Study. Blood Press Monit. 2006;11(4):229-34.

23. Matsudo S, Araújo T, Matsudo V, Andrade D, Andrade E, Oliveira LC, et al Questionário internacional de atividade física (Ipaq): estupo de validade e reprodutibilidade no Brasil. Rev Bras Ativ Fis Saúde. 2012;6(2):5-18.

24. Lo K, Wong M, Khalechelvam P, Tam W. Waist-to-height ratio, body mass index and waist circumference for screening paediatric cardio-metabolic risk factors: a meta-analysis. Obes Rev. 2016;17(12):1258-75.

25. Rodrigues S, Baldo M, Mill JG. Association of waist-stature ratio with hypertension and metabolic syndrome: population-based study. Arq Bras Cardiol. 2010;95(2):186-91

26. Gleeson M, Bishop NC, Stensel DJ, Lindley MR, Mastana SS, Nimmo MA. The anti-inflammatory effects of exercise: mechanisms and implications for the prevention and treatment of disease. Nat Rev Immunol. 2011;11(9):607-15.

27. Seravalle G, Grassi G. Obesity and hypertension. Pharmacol Res. 2017 Aug;122:1-7.

28. Brasil.Ministério da Saúde. Secretaria Municipal da Saúde. Centro de Epidemiologia. Coordenação de Vigilância Nutricional. SISVAN - Sistema de Vigilância Alimentar e Nutricional do Escolar. Brasilia; 2017.

29. Tarp J, Child A, White T, Westgate K, Bugge A, Grontved A, et al. Physical activity intensity, bout-duration, and cardiometabolic risk markers in children and adolescents. Int J Obes. 2018;42(9):1639-50.

30. Sadoh WE, Sadoh AE, Onyiriuka AN. Physical activity, body mass index and blood pressure in primary school pupils attending private schools. Afr Health Sci. 2016;16(4):947-53

31. Corrêa-Neto VG, Sperandei S, Silva LAI, Maranhão-Neto GA, Palma A. Arterial hypertension among adolescents in Rio de Janeiro: prevalence and association with physical activity and obesity. Cien Saúde Colet. 2014;19(6):1699-708.

32. Kort-Butler LA, Hagewen KJ. School-based extracurricular activity involvement and adolescent self-esteem: a growth-curve analysis. J Youth Adolesc. 2011;40(5):568-81.

33. Craemer M, Decker E, Bourdeaudhuij I, Vereecken C, Deforche B, Manios Y, et al. Correlates of energy balance-related behaviours in preschool children: a systematic review. Obes Rev. 2012;13(Suppl 1):13-28.

34. World Health Organization. Global Recommendations on Physical Activity for Health. Geneva: WHO; 2010.

35. Agostinis-Sobrinho C, Ruiz JR, Moreira C, Lopes L, Ramírez-Vélez R, GarcíaHermoso A, et al. Changes in muscular fitness and its association with blood pressure in adolescents. Eur J Pediatr. 2018;177(7):1101-9.

36. Cureau FV, Silva TLN, Bloch KV, Fujimori E, Belfort DR, Carvalho KMB, et al ERICA: leisure-time physical inactivity in Brazilian adolescents. Rev Saúde Publica. 2016;50(Suppl 1):1s-11s. 